\title{
Antibiotic trends in acute febrile illness
}

\author{
Riyaz Ahmed Siddiqui*, Tanaji R. Shende, Y. Dhoble, Archana S. Borkar
}

Department of Pharmacology, NKPSIMS, Nagpur,

Maharashtra, India

Received: 29 November 2016

Accepted: 24 December 2016

\section{*Correspondence to:}

Dr. Riyaz Ahmed Siddiqui, Email:

riyaz_ammar@rediffmail.com

Copyright: (C) the author(s), publisher and licensee Medip Academy. This is an openaccess article distributed under the terms of the Creative Commons Attribution NonCommercial License, which permits unrestricted noncommercial use, distribution, and reproduction in any medium, provided the original work is properly cited.

\begin{abstract}
Background: Acute febrile illness has various etiologies. Different antimicrobials are used for different causes of fever to treat and there are interpersonal variations in prescription of antimicrobials. Therefore this study aims at to analyze the trends in the antimicrobial prescription in patients of acute febrile illness due to various etiologies in the medicine department of a tertiary care hospital.
\end{abstract}

Methods: It is a record based observational study that was carried out at NKP salve institute of medical sciences and RC, Nagpur. 200 case record files of patients admitted with a diagnosis of acute febrile illness in the dept. of Medicine due to various etiologies were analyzed. Antibiotics prescribed for various causes of acute febrile illness like respiratory tract infections, urinary tract infection, gastrointestinal infection, malarial infections, septicemia, meningitis, pyrexia of unknown origin etc. were noted and data was analyzed for prescription pattern of antimicrobials.

Results: Out of 200 patients of febrile illness the common clinical conditions for which antibiotics were prescribed were respiratory tract infections (upper respiratory tract infection $25.5 \%$ and lower respiratory tract infection $14 \%$ ), acute gastroenteritis $(20 \%)$, and urinary tract infection $(13.5 \%)$ followed by, pyrexia of unknown origin $(8.5 \%)$, viral fever $(8 \%)$, malaria $(7 \%)$, hepatitis A $(1.5 \%)$, meningitis $(1 \%)$ and rickettsial infection (1\%). The commonly prescribed antibiotics were ceftriaxone in $(19.37 \%)$ and cefixime $(15.93 \%)$ followed by coamoxiclav (12.5\%), azithromycin (11.87\%), doxycycline $(10.31 \%)$, ofloxacin and ornidazole $(8.43 \%)$, levofloxacin $(6.25 \%)$, ofloxacin $(4.68 \%)$, ciprofloxacin (1.87\%), artesunate $(3.75 \%)$, artemether and lumefantrine $(4.37 \%)$ and valacyclovir $(0.625 \%)$.

Conclusions: Our study concluded that most common disease for which antibiotics prescribed were respiratory tract infection and gasterointestinal infections. Most common antibiotic used were third generation cephalosporins especially ceftriaxone and cefixime.

Keywords: Anti microbial agents, Gastrointestinal infections, Respiratory tract infection

\section{INTRODUCTION}

"Acute febrile illness", or "acute fever" or "short febrile illness" is traditionally defined as any illness associated with fever of two weeks or shorter in duration, rapid in onset, caused by diverse pathogens. The clinical signs and symptoms of most of these infections are very similar and the correct diagnosis is only possible by using pathogen specific diagnostic tests. Several studies have documented the etiology of acute febrile illness in tropical countries like India as Dengue, Malaria, Typhoid fever, Leptospirosis, Rickettsial infection. ${ }^{1}$ The etiologies of human febrile illness can vary region wise in India suggesting that diagnosis, treatment, and control programs need to be based on a methodical evaluation of area specific etiologies. Knowledge of local prevalence of infections is critical in order to target clinical work up and treatment. The various common causes of acute febrile illness in tropical countries have similar clinical presentation. A large number of patients present to Indian hospitals with acute febrile illness and multisystem involvement. There may be overlapping clinical 
presentation in various acute febrile illnesses and it is important to diagnose the specific etiology so that appropriate treatment can be initiated. Disease burden of infectious etiologies of acute febrile illness is under reported in various parts of India due to lack of laboratory confirmation. Undifferentiated febrile illnesses are common in tropical areas of Asia. Common causes include dengue, malaria, leptospirosis, enteric fever, chikungunya, rickettsia and Japanese encephalitis. ${ }^{2}$ Further confounding this is the fact that a majority of the patients present with non-descript symptoms (e.g., lowgrade fever, general malaise, headache, and myalgia) and usually no focal point of infection. Health care providers lacking proper diagnostic tools are usually unable to determine specific etiologies, often diagnosing patients presumptively based on clinical features and assumptions regarding circulating pathogens. Syndrome based disease surveillance provides a useful methodology to systematically identify and document causes of acute fever. ${ }^{3}$

Drug use studies using aggregate data indicate that there is over or under consumption of medicines. The data on utilization may provide useful information for promoting appropriate use of medicines. Antimicrobial agents are among the most frequently prescribed drugs. Inappropriate use of these agents is associated with allergic reactions, toxicity, super infection, and more importantly the development of antimicrobial resistance. In addition, the excessive and inappropriate use of antimicrobials can cause an unnecessary economic burden to health care system and the patients as well. Antimicrobial resistance is more prevalent in hospital settings than in the community. Studies have shown that patients with drug-resistant organisms require longer hospitalization and have increased risk of mortality. Inappropriate prescribing is a recognized worldwide problem of the health care delivery system. In recent years, drug utilization studies are found to be useful tool to facilitate rational use of drugs in health care delivery systems. It truly reflects the status of health care system. In order to be rational use of a drug must be effective, safe, prescribed for the proper therapeutic indication and the correct dosage in an appropriate formulation, easily available and of a reasonable cost. ${ }^{5}$

Paediatric population is prone to suffer from recurrent infections of the respiratory tract and gastrointestinal system. Lower respiratory tract infections are the leading cause of death in children below 5 five years of age. Acute respiratory infection, acute watery diarrhoea and viral fever are the common childhood illnesses accounting for the major proportion of paediatric visits. ${ }^{6}$ Several studies have reported that $50 \%$ to $85 \%$ of children receive antibiotics in developed and developing countries. In case of prescribing for children, there is a need to consider the etiopathogenesis of the disease conditions as well as the developmental stage of the child at that particular time. ${ }^{7}$ Drug utilization study is a great tool for analysing and evaluating prescribing pattern of medical professionals and also helps in formulating drug and antibiotics policy. ${ }^{8}$ Until now very few studies are conducted in this field. Therefore this study is undertaken to analyze the trends in the antimicrobial prescription in patients of acute febrile illness due to various etiologies in the medicine department of a tertiary care hospital.

\section{METHODS}

It is a record based observational study that was carried out at NKP salve institute of medical sciences and RC, Nagpur. Ethical committee permission was obtained prior to the study. In this study we have analyzed 200 case record files of patients admitted with a diagnosis of acute febrile illness in the dept. of Medicine due to various etiologies. Antibiotics prescribed for various causes of acute febrile illness like respiratory tract infections, urinary tract infection, gastrointestinal infection, malarial infections, septicemia, meningitis, pyrexia of unknown origin etc. were be noted. Data collected was analyzed for proportion.

\section{RESULTS}

A total of 200 patients who were prescribed antimicrobial agents (AMA) were analysed. The common clinical conditions for which antibiotics were prescribed are respiratory tract infections (upper respiratory tract infection $25.5 \%$ and lower respiratory tract infection $14 \%)$, acute gastroenteritis (20\%), and urinary tract infection $(13.5 \%)$ followed by, pyrexia of unknown origin $(8.5 \%)$, viral fever $(8 \%)$, malaria $(7 \%)$, hepatitis A $(1.5 \%)$, meningitis $(1 \%)$ and rickettsial infection $(1 \%)$ as shown in Table 1.

Table 1: Various causes antimicrobial agents prescription.

\begin{tabular}{|lll|}
\hline Disease & No. of patients & Percentage \\
\hline URTI & 51 & 25.5 \\
\hline LRTI & 28 & 14 \\
\hline Acute gastroenteritis & 40 & 20 \\
\hline Urinary tract infection & 27 & 13.5 \\
\hline PUO & 17 & 8.5 \\
\hline Viral fever & 16 & 8 \\
\hline Malaria & 14 & 7 \\
\hline Hepatitis A & 3 & 1.5 \\
\hline Meningitis & 2 & 1 \\
\hline Rickettsial infection & 2 & 1 \\
\hline
\end{tabular}

The commonly prescribed antibiotics were ceftriaxone in $(19.37 \%)$ and cefixime $(15.93 \%)$ followed by coamoxiclav (12.5\%), Azithromycin (11.87\%), Doxycycline (10.31\%), ofloxacin and ornidazole $(8.43 \%)$, levofloxacin (6.25\%), ofloxacin (4.68\%), ciprofloxacin $(1.87 \%)$, artesunate $(3.75 \%)$, artemether and lumafanterene $(4.37 \%)$ and valacyclovir $(0.625 \%)$ as shown in Table 2. 
Table 2: Commonly prescribed antimicrobial agents (AMA).

\begin{tabular}{|lll|}
\hline $\begin{array}{l}\text { Antimicrobial } \\
\text { agents (AMA) }\end{array}$ & No. of patients & Percentage \\
\hline Ceftriaxone & 62 & 19.37 \\
\hline Cefixime & 51 & 15.93 \\
\hline Coamoxyclav & 40 & 12.5 \\
\hline Azithromycin & 38 & 11.87 \\
\hline Doxycycline & 33 & 10.31 \\
\hline $\begin{array}{l}\text { Ofloxacin and } \\
\text { ornidazole }\end{array}$ & 27 & 8.43 \\
\hline Levofloxacin & 20 & 6.25 \\
\hline Ofloxacin & 15 & 4.68 \\
\hline Ciprofloxacin & 6 & 1.87 \\
\hline Artesunate & 12 & 3.75 \\
\hline $\begin{array}{l}\text { Artemether and } \\
\text { lumefantrine }\end{array}$ & 14 & 4.37 \\
\hline Valacyclovir & 2 & 0.625 \\
\hline
\end{tabular}

\section{DISCUSSION}

We have studied a total of 200 patients who were prescribed anti microbial agents (AMA) for febrile illness. The most common clinical conditions for which antibiotics were prescribed are respiratory tract infections (upper respiratory tract infection $25.5 \%$ and lower respiratory tract infection $14 \%$ ), acute gastroenteritis $(20 \%)$, and urinary tract infection $(13.5 \%)$ followed by, pyrexia of unknown origin $(8.5 \%)$, viral fever $(8 \%)$, malaria (7\%), hepatitis A $(1.5 \%)$, meningitis $(1 \%)$ and rickettsial infection (1\%). Commonly prescribed antibiotics were ceftriaxone in $(19.37 \%)$ and cefixime $(15.93 \%)$ followed by coamoxiclav (12.5\%), azithromycin $(11.87 \%)$, doxycycline $(10.31 \%)$, ofloxacin and ornidazole $(8.43 \%)$, levofloxacin $(6.25 \%)$, ofloxacin (4.68\%), ciprofloxacin (1.87\%), artesunate (3.75\%), artemether and lumefantrine $(4.37 \%)$ and valacyclovir $(0.625 \%)$. In a study conducted by Meher et al the common clinical conditions for which antibiotics were prescribed are Respiratory tract infection (24\%), Urinary tract infection (18\%), Gastroenteritis (18\%) Typhoid fever (16\%), Septicemia (13\%) Meningitis (8\%) Pyrexia of unknown origin (3\%), and the commonly prescribed antibiotics were ceftriaxone $(30.03 \%)$, coamoxiclav (22.6\%), amikacin (16.33\%), ciproploxacin (13.41\%), metronidazole (12.34\%), Levoflocacin $(5.09 \%){ }^{8}$ Ahmad et al found that Respiratory tract infection was the most common condition for which antibiotics were prescribed in $(21.5 \%)$, followed by viral fever $(12 \%)$, UTI $(8.5 \%)$, and acute gastroenteritis $(7.5 \%)$. The most common antibiotics prescribed were cephalosporins (62.5\%) followed by fluroquinolones $(16.5 \%)$, penicillin $(16 \%)$, Nitroimidazole (14\%) and Amikacin (10\%). Ceftriaxone was the most commonly used cephalosporin antibiotic. ${ }^{9}$ In a retrospective study carried out in patients who received at least one antibiotic in General medicine department, the antibiotics were mainly prescribed for COPD, Pneumonia, LRTI, UTI, PUD, URTI, and Viral fever, ALD, Tuberculosis, Acute Bacillary Dysentery, GERD, Rheumatoid Arthritis and Surgery. Majority of the patients were prescribed with cephalosporin category of antibiotics, followed by fluoroquinolones, penicillin and aminoglycosides. ${ }^{10}$ Antibiotics are considered among the most commonly prescribed drug classes in developing countries. Inappropriate prescription of antibiotics is a major public health concern and is related to the development of antimicrobial resistance. Saleh et al in a cross sectional study of 270 patients $(49.3 \%$ males and $50.7 \%$ females) showed that the most-prescribed antibiotics were the cephalosporins $82 \%$ (mainly cefixime, an oral third-generation cephalosporin) and that almost half of the illnesses for which antibiotics were prescribed were respiratory tract infections $(41 \%){ }^{11}$ Kanish et al studied patients admitted in the pediatric ward and ICU in a tertiary care hospital, found that beta lactams (cephalosporins the most) were maximally prescribed followed by aminoglycosides. Third generation cephalosporins were prescribed more frequently since it was a tertiary care hospital and patients would have already been administered and probably developed resistance to lower generation antibiotics. ${ }^{12}$ The above studies are in accordance with our study in which respiratory tract infections are the commonest cause of antibiotic use and cephalosporins especially ceftriaxone and cefixime are the commonly prescribed antibiotics.

Chandy et al carried out study of Surveillance of antibiotic encounters (prescriptions and dispensations) for 2 years and found that fluoroquinolones and penicillins were widely used. Rural hospitals used co-trimoxazole more often and urban private hospitals used cephalosporins more often; $41.1 \%$ of antibiotic prescriptions were for respiratory infection. ${ }^{13}$ Pasha et al in their study reviewed 250 cases to evaluate the prescribing pattern of antibiotics in the treatment of various types of infection found that majority of the patients were treated with Quinolones (36.55\%), the major route of drug administration employed was oral route. The most common were ciprofloxacin, amoxicillin, metronidazole, ampicillin, aminoglycosides, macrolide antibiotics, cephalosporin and sulphonamides. ${ }^{14}$ In the above studies fluoroquinolones were the commonly used antimicrobials which is not in accordance with our study. This could be due to interpersonal variation in prescription of antimicrobials Bansal et al study seven hundred thirty patients who were screened and 550 enrolled, receiving 1,512 courses of antimicrobial therapy, mainly intravenously (66\%). Most frequently prescribed agents were artesunate (13\%), ceftriaxone $(11 \%)$ and metronidazole $(10.5 \%) .^{15}$

\section{CONCLUSION}

Different antibiotics are prescribed for different etiologies of febrile illness. Our study concluded that most common disease for which antibiotics prescribed were respiratory tract infection and gastrointestinal infections. Most 
common antibiotic used were third generation cephalosporins especially ceftriaxone and cefixime.The study of prescribing pattern is a part of the medical audit and seeks to monitor, evaluate and if necessary, suggest modification in prescribing practices to make medical care rational and cost effective.

Funding: No funding sources

Conflict of interest: None declared

Ethical approval: The study was approved by the Institutional Ethics Committee

\section{REFERENCES}

1. Rani RA, Sundararajan T, Rajesh S, Jeyamurugan T. A Study on Common Etiologies of Acute Febrile Illness Detectable by Microbiological Tests in a Tertiary Care Hospital. Int. J. Curr. Microbiol. App. Sci. 2016;5(7):670-4.

2. Das D, Das B, Roy AD, Singh TSK. Common Infectious Etiologies of Acute Febrile Illness in a Remote Geographical Location: Could Scrub Typhus be the Most Common Cause? British Journal of Medicine and Medical Research. 2015;10(10):1-10.

3. Kashinkunti MD, Gundikeri SK, Dhananjaya M. Acute undifferentiated febrile illness- clinical spectrum and outcome from a ertiary care teaching hospital of north Karnataka. Int J Biol Med Res. 2013;4(2):3399-402.

4. Khety Z, Mohanta GP, Patvardhan S, Jain S. Studies on Antimicrobial Consumption in a Tertiary Care Private Hospital, India. IJPPR. 2016;6(1).

5. Reddy SN, Manohar B, Bhavani DM, Kejiya G, Sreevidya C, Ranganayakulu D. Prescribing pattern of drugs in pediatric wards at tertiary care teaching hospital. International Journal of Pharmacy Review and Research. 2015;5(4):374-8.

6. Venkateswaramurthy N, Murali R, Kumar RS. The study of drug utilization pattern in pediatric patients. International Journal of Pharmacy and Pharmaceutical Sciences. 2013;5(3).

7. Narayan DS, Mangesh MM. A Study of Prescription Pattern of Antibiotics in Paediatric In-Patients at a tertiary care hospital in central India IJPR. 2016;6(8).
8. Meher BR, Mukharjee D, Udayshankar. A study on antibiotic utilization pattern in a general medicine ward of a tertiary care teaching hospital. Journal of Chemical and Pharmaceutical Research. 2014;6(7):1847-9.

9. Ahmad A, Revanker M, Haque I, Pravina A, Ivan R, Dasari R, et al. Study the Prescription Pattern of Antibiotics in the Medicine Department in a Teaching Hospital: A Descriptive Study. International Journal of Toxicological and Pharmacological Research. 2014;6(2):43-6.

10. Chitra B. Study on utilization pattern of antibiotics at a private corporate hospital. Indian Journal of Drugs. 2016;4(3):69-74

11. Saleh N, Awada S, Awwad R, Jibai S, Arfoul C, Zaiter $\mathrm{L}$, et al. evaluation of antibiotic prescription in the lebanese community: a pilot study. Infection ecology and epidemiology; 2015;5.

12. Kanish R, Gupta K, Juneja S, Bains HS, Kaushal S. Prescribing pattern and pharmacoeconomics of antibiotic use in the department of pediatrics of a tertiary care medical college hospital in northern India. Annals of tropical medicine and public health. 2015;8(4):101-4.

13. Chandy SJ, Thomas K, Mathai E, Antonisamy B, Kathleen A. Holloway 6 and Cecilia Stals by Lundborg Patterns of antibiotic use in the community and challenges of antibiotic surveillance in a lowermiddle-income country setting: A repeated crosssectional study in Vellore, south India. J. Antimicrob. Chemother. 2013;68(1):229-36.

14. Pasha SZ, Prasad BD, Shalem L, Chand TB, Veeramani G. Prescribing pattern and use of antibiotics and combination of antibiotics in a tertiary care teaching hospital. IAJPR. 2014;4(7):3172-81.

15. Bansal D, Mangla S, Undela K, Gudala K, D’Cruz S, Sachdev A, et al. Measurement of Adult Antimicrobial Drug Use in Tertiary Care Hospital Using Defined Daily Dose and Days of Therapy. Indian J Pharm Sci. 2014;76(3):211-7.

Cite this article as: Siddiqui RA, Shende TR, Dhoble Y, Borkar AS. Antibiotic trends in acute febrile illness. Int J Basic Clin Pharmacol 2017;6:334-7. 